\title{
On the Floquet Exponents of Hill's Equation Systems
}

\author{
By Robert Denk of Regensburg
}

\begin{abstract}
For the $k \times k$-matrix-valued version of Hill's equation it is shown that the dimension of the matrix needed to compute the Floquet exponents can be reduced from $2 k$ to $k$. Also the existence of periodic solutions is equivalent to the non-invertibility of certain $k \times k$-matrices.
\end{abstract}

\section{Introduction}

In this paper we consider a matrix-valued version of Hill's equation, i.e. the following second order system of ordinary differential equations

$$
y^{\prime \prime}(x)+G(x) \cdot y(x)=0 \quad(x \in \mathbb{R}) .
$$

Here $G$ is an even $k \times k$-matrix-valued function with period $T$. We assume that the entries of $G$ are Lebesgue-integrable on the interval $[0, T]$.

In the case where $k$ equals 1 equation (1) was investigated first by HILL [5] in 1886. Since then the scalar form of (1) has been an important field of research, for results concerning this case the reader is referred to the book of MAgnus and WinkLER [7]. The matrix case where $k$ is an arbitrary positive integer, however, can hardly be found in literature. Whereas the general theory of linear ODE systems with periodic coefficients can be applied (this theory is described in the book of YaKUBOVICH and STARzHINSKII [12]), the symmetry properties of (1) allow us to obtain more specific results. For applications of equation (1) or some generalized form of it to the mechanics of gearings see, for instance, [1], [11].

Due to the periodicity of the function $G$, the theory of Floquet can be applied. The famous theorem of Floquet-Lyapunov (Theorem 2 below) tells us that stability properties and existence of periodic solutions of (1) are connected with the so-called Floquet exponents (characteristic exponents) of this equation. These are defined as the complex numbers $\nu$ where a solution $y(x)$ of (1) exists with $y(x+T)=e^{i T \nu} y(x)$. According to the FloquetLyapunov theorem the Floquet exponents can be calculated as the eigenvalues of a $2 k \times 2 k$ matrix.

In Section 2 of this paper we show that it is sufficient to consider the eigenvalues of a $k \times k$-matrix (Theorem 3 ). The proof of this theorem uses the symmetry of the function $G$ 
and bases on a comparison of the eigenvalues of the matrix solutions of (1) which form the matrizant of this equation. The main part of the proof is done in Lemma 1. In Section 3 we consider the question of periodic or antiperiodic solutions of equation (1). Theorem 4 shows some factorizations for the determinants of the corresponding matrix solutions. As a corollary of this theorem, the existence of periodic/antiperiodic solutions can be reduced to the question if the matrix solutions are nonsingular at the point $\frac{T}{2}$.

This paper contains some part of the author's thesis [3], the results in sections 2 and 3 are generalizations of the classical results in the case $k=1$ (cf. [7], [8]).

\section{Floquet exponents and fundamental solutions}

In the following $\mathrm{M}(k \times k, \mathbb{C})$ stands for the set of all $k \times k$-matrices with complex coefficients. Let $Y_{1,2}: \mathbb{R} \rightarrow \mathrm{M}(k \times k, \mathbb{C})$ be the matrix solutions of system (1) with the initial values

$$
\begin{array}{ll}
Y_{1}(0)=I_{k}, & Y_{2}(0)=0, \\
Y_{1}^{\prime}(0)=0, & Y_{2}^{\prime}(0)=I_{k},
\end{array}
$$

where $I_{k}$ denotes the unit matrix. The fundamental solution or matrizant of (1) is defined by

$$
Y(x):=\left(\begin{array}{cc}
Y_{1}(x) & Y_{2}(x) \\
Y_{1}^{\prime}(x) & Y_{2}^{\prime}(x)
\end{array}\right)
$$

We first mention some properties of $Y(x)$ (cf. [4, V.1], [12, II.3.9]):

\section{Theorem 1.}

a) For all $x \in \mathbb{R}$ we have $\operatorname{det} Y(x)=1$.

b) Let $H:=\left(\begin{array}{cc}-I_{k} & 0 \\ 0 & I_{k}\end{array}\right)$. Then $Y(x)=H Y(-x) H$ and $Y(x \pm T)=Y(x) Y( \pm T)$. In particular we have $Y(-T)=Y(T)^{-1}$ and

$$
Y\left(\frac{T}{2}\right)=Y\left(-\frac{T}{2}\right) Y(T) .
$$

The following theorem summarizes some results of Floquet theory (cf. [2], [9], [12]) concerning the stability and the existence of periodic/antiperiodic solutions of (1). (A solution $y(x)$ is called $T$-antiperiodic if $y(x+T)=-y(x)$ for all $x \in \mathbb{R}$.)

\section{Theorem 2.}

a) $\nu$ is a Floquet exponent of (1) if and only if $e^{i T \nu}$ is an eigenvalue of $Y(T)$.

b) There exists a T-periodic [T-antiperiodic] solution of (1) if and only if $\nu=0\left[\nu=\frac{\pi}{T}\right]$ is a Floquet exponent of (1).

c) All solutions of (1) are bounded if and only if the following condition holds: All Floquet exponents are real and for every eigenvalue of $Y(T)$ the algebraic and geometric multiplicities are equal. 
Remark 1. Theorem 2a) enables us to calculate the Floquet exponents by numerical integration on the interval $[0, T]$. From part b) of Theorem 1 we see that

$$
Y(T)=H Y\left(\frac{T}{2}\right)^{-1} H Y\left(\frac{T}{2}\right)
$$

Therefore the Floquet exponents of (1) can be computed by numerical integration on the interval $\left[0, \frac{T}{2}\right]$.

For the next theorem we need the following auxiliary result (here tr denotes the trace of a matrix).

\section{Lemma 1.}

a) For all $n \in \mathbb{N}$ we have $\operatorname{tr}\left(Y_{1}(T)^{n}\right)=\operatorname{tr}\left(Y_{2}^{\prime}(T)^{n}\right)$.

b) $Y_{1}(T)$ and $Y_{2}^{\prime}(T)$ have the same eigenvalues with the same algebraic multiplicities.

Proof. a) From (3) it follows that $Y(T) H$ is similar to $H$. Therefore $\operatorname{tr}(Y(T) H)=0$ and the stated equality for $n=1$ follows.

Let $n=2 m(m \in \mathbb{N})$. According to $Y(T)^{-1}=Y(-T)=H Y(T) H$ we have

$$
Y_{1}(T)^{2}-Y_{2}(T) Y_{1}^{\prime}(T)=I_{k}
$$

and

$$
Y_{2}^{\prime}(T)^{2}-Y_{1}^{\prime}(T) Y_{2}(T)=I_{k}
$$

Substituting these expressions for $Y_{1}(T)^{2}$ and $Y_{2}^{\prime}(T)^{2}$, using the linearity of the trace function and the fact that $\operatorname{tr}(M N)=\operatorname{tr}(N M)$ for matrices $M, N \in \mathrm{M}(k \times k, \mathbb{C})$, we can write

$$
\begin{aligned}
\operatorname{tr}\left(Y_{1}(T)^{2 m}\right) & =\operatorname{tr}\left[\left(I_{k}+Y_{2}(T) Y_{1}^{\prime}(T)\right)^{m}\right]=\sum_{j=0}^{m}\left(\begin{array}{c}
m \\
j
\end{array}\right) \operatorname{tr}\left[\left(Y_{2}(T) Y_{1}^{\prime}(T)\right)^{j}\right]= \\
& =\operatorname{tr}\left[\left(I_{k}+Y_{1}^{\prime}(T) Y_{2}(T)\right)^{m}\right]=\operatorname{tr}\left(Y_{2}^{\prime}(T)^{2 m}\right) .
\end{aligned}
$$

If $n=2 m+1(m \in \mathbb{N})$, we get

$$
\begin{aligned}
\operatorname{tr}\left(Y_{1}(T)^{2 m+1}\right) & =\sum_{j=0}^{m}\left(\begin{array}{c}
m \\
j
\end{array}\right) \operatorname{tr}\left[Y_{1}(T)\left(Y_{2}(T) Y_{1}^{\prime}(T)\right)^{j}\right]= \\
& =\sum_{j=0}^{m}\left(\begin{array}{c}
m \\
j
\end{array}\right) \operatorname{tr}\left[Y_{2}^{\prime}(T)\left(Y_{1}^{\prime}(T) Y_{2}(T)\right)^{j}\right]=\operatorname{tr}\left(Y_{2}^{\prime}(T)^{2 m+1}\right)
\end{aligned}
$$

where the second equality follows from the case $n=1$ for $j=0$ and from

$$
Y_{1}(T) Y_{2}(T)=Y_{2}(T) Y_{2}^{\prime}(T)
$$

(cf. Theorem $1 \mathrm{~b})$ ) for $j>0$.

b) If we write

$$
\operatorname{det}\left(M-z I_{k}\right)=\sum_{j=0}^{k} \alpha_{j}(M) z^{k-j} \text { for } M \in \mathrm{M}(k \times k, \mathbb{C})
$$


the following identity holds [6, p. 117]:

$$
\alpha_{j}(M)=\frac{(-1)^{j}}{j !} \operatorname{det}\left(\begin{array}{ccccc}
\sigma_{1} & 1 & 0 & \cdots & 0 \\
\sigma_{2} & \sigma_{1} & 2 & & 0 \\
\vdots & \vdots & & \ddots & \vdots \\
\sigma_{j-1} & \sigma_{j-2} & \ddots & j-1 \\
\sigma_{j} & \sigma_{j-1} & & \cdots & \sigma_{1}
\end{array}\right),
$$

with $\sigma_{j}:=\operatorname{tr}\left(M^{j}\right)$. From this and a) we see that

$$
\operatorname{det}\left(Y_{1}(T)-z I_{k}\right)=\operatorname{det}\left(Y_{2}^{\prime}(T)-z I_{k}\right) \text {. }
$$

Remark 2. a) If one of the matrices $Y_{1}^{\prime}(T)$ or $Y_{2}(T)$ is non-singular, the result of Lemma $1 \mathrm{~b}$ ) is an immediate consequence of Theorem $1 \mathrm{~b}$ ).

b) In the scalar case $k=1$ we get the well-known equality $Y_{1}(T)=Y_{2}^{\prime}(T)$ (cf. [8, 2.12]).

Theorem 3. $\nu$ is a Floquet exponent of (1) if and only if $\cos (T \nu)$ is an eigenvalue of $Y_{1}(T)$. More precisely, we have

$$
\operatorname{det}\left(Y(T)-e^{i T \nu} \cdot I_{2 k}\right)=(-2)^{k} e^{i T \nu k} \operatorname{det}\left(Y_{1}(T)-\cos (T \nu) \cdot I_{k}\right) .
$$

Proof. If $\lambda:=e^{i T \nu}$ is no eigenvalue of $Y_{1}(T)$, Lemma $\left.1 \mathrm{~b}\right)$ tells us that also $Y_{2}^{\prime}(T)-\lambda I_{k}$ is nonsingular and we can write

$$
\begin{aligned}
& \operatorname{det}\left(Y(T)-\lambda I_{2 k}\right)= \\
& \quad=\operatorname{det}\left[Y_{2}^{\prime}(T)-\lambda I_{k}\right] \operatorname{det}\left[\left(Y_{1}(T)-\lambda I_{k}\right)-Y_{2}(T)\left(Y_{2}^{\prime}(T)-\lambda I_{k}\right)^{-1} Y_{1}^{\prime}(T)\right] .
\end{aligned}
$$

From $Y(T)^{-1}=Y(-T)$, making some elementary calculations, we obtain

$$
\left(Y_{1}(T)^{2}-I_{k}\right)\left(Y_{1}(T)-\lambda I_{k}\right)^{-1}=Y_{2}(T)\left(Y_{2}^{\prime}(T)-\lambda I_{k}\right)^{-1} Y_{1}^{\prime}(T) .
$$

With (5) and Lemma 1 b) we get

$$
\begin{aligned}
& \operatorname{det}\left(Y(T)-\lambda I_{2 k}\right)= \\
& =\operatorname{det}\left[Y_{2}^{\prime}(T)-\lambda I_{k}\right] \operatorname{det}\left[\left(Y_{1}(T)-\lambda I_{k}\right)-\left(Y_{1}(T)^{2}-I_{k}\right)\left(Y_{1}(T)-\lambda I_{k}\right)^{-1}\right]= \\
& =\operatorname{det}\left[\left(\lambda^{2}+1\right) I_{k}-2 \lambda Y_{1}(T)\right]= \\
& =\operatorname{det}\left[2 e^{i T \nu}\left(\left(\frac{1}{2} e^{i T \nu}+\frac{1}{2} e^{-i T \nu}\right) I_{k}-Y_{1}(T)\right)\right] \\
& =(-2)^{k} e^{i T \nu k} \operatorname{det}\left[Y_{1}(T)-\cos (T \nu) \cdot I_{k}\right] .
\end{aligned}
$$

As both sides of (4) are holomorphic in $\nu$, we get the theorem for arbitrary values of $\nu \in \mathbb{C}$.

The preceeding theorem is a generalization of the condition $\cos (T \nu)=Y_{1}(T)$ in the case $k=1[8,2.13]$. In that case the proof is much simpler and uses only the equalities

$$
Y_{1}(T)=Y_{2}^{\prime}(T) \text { and } Y_{1}^{\prime}(T) Y_{2}(T)=Y_{1}(T)^{2}+1 \text {. }
$$

The first of these equalities, for instance, is an immediate consequence of the case $n=1$ in Lemma 1 a). 


\section{Existence of periodic solutions}

Due to Floquet's theory, there exists a solution $y$ of (2) with period $T$ if and only if 0 is a Floquet exponent, i.e. if and only if 1 is an eigenvalue of $Y_{1}(T)$. We now show that the existence of periodic solutions can be checked by the determinants of the fundamental solutions $Y_{1}(x), Y_{2}(x)$ and their derivatives for $x=\frac{T}{2}$. This is based on Theorem 4 that shows some factorizations not for the fundamental solutions themselves, but for their determinants. As we will see in Lemma 2, more specific statements can be made concerning the existence of even/odd solutions with period $T$.

Theorem 4. We have the following factorizations:

(i) $\operatorname{det}\left(Y_{1}(T)-I_{k}\right)=2^{k} \operatorname{det} Y_{1}^{\prime}\left(\frac{T}{2}\right) \operatorname{det} Y_{2}\left(\frac{T}{2}\right)$,

(ii) $\operatorname{det}\left(Y_{1}(T)+I_{k}\right)=2^{k} \operatorname{det} Y_{1}\left(\frac{T}{2}\right) \operatorname{det} Y_{2}^{\prime}\left(\frac{T}{2}\right)$,

(iii) $\quad \operatorname{det} Y_{1}^{\prime}(T)=2^{k} \operatorname{det} Y_{1}\left(\frac{T}{2}\right) \operatorname{det} Y_{1}^{\prime}\left(\frac{T}{2}\right)$,

(iv) $\quad \operatorname{det} Y_{2}(T)=2^{k} \operatorname{det} Y_{2}\left(\frac{T}{2}\right) \operatorname{det} Y_{2}^{\prime}\left(\frac{T}{2}\right)$.

Proof. We write $Y\left(\frac{T}{2}\right)^{-1}=:\left(\begin{array}{cc}Z_{11} & Z_{12} \\ Z_{21} & Z_{22}\end{array}\right)$. First we show that

$$
\operatorname{det} Z_{12}=(-1)^{k} \cdot \operatorname{det} Y_{2}\left(\frac{T}{2}\right) \text {. }
$$

If $\operatorname{det} Y_{2}\left(\frac{T}{2}\right) \neq 0$ we have

$Y\left(\frac{T}{2}\right)=\left[\begin{array}{cc}Y_{2}\left(\frac{T}{2}\right) & 0 \\ Y_{2}^{\prime}\left(\frac{T}{2}\right) & I_{k}\end{array}\right]\left[\begin{array}{cc}I_{k} & -Y_{2}\left(\frac{T}{2}\right)^{-1} Y_{1}\left(\frac{T}{2}\right) \\ 0 & -Y_{1}^{\prime}\left(\frac{T}{2}\right)+Y_{2}^{\prime}\left(\frac{T}{2}\right) Y_{2}\left(\frac{T}{2}\right)^{-1} Y_{1}\left(\frac{T}{2}\right)\end{array}\right]\left[\begin{array}{cc}0 & I_{k} \\ -I_{k} & 0\end{array}\right]$.

With Theorem 1a) we see that

$$
\operatorname{det} Y_{2}\left(\frac{T}{2}\right)=\operatorname{det}\left(-Y_{1}^{\prime}\left(\frac{T}{2}\right)+Y_{2}^{\prime}\left(\frac{T}{2}\right) Y_{2}\left(\frac{T}{2}\right)^{-1} Y_{1}\left(\frac{T}{2}\right)\right)^{-1} .
$$

Inverting both sides of (7) yields

$$
-Z_{12}=\left(-Y_{1}^{\prime}\left(\frac{T}{2}\right)+Y_{2}^{\prime}\left(\frac{T}{2}\right) Y_{2}\left(\frac{T}{2}\right)^{-1} Y_{1}\left(\frac{T}{2}\right)\right)^{-1}
$$

and therefore (6). 
Now let $\operatorname{det} Y_{2}\left(\frac{T}{2}\right)=0$. If we assume $\operatorname{det} Z_{12} \neq 0$, a similar calculation shows

$$
\left(Z_{21}-Z_{22} Z_{12}^{-1} Z_{11}\right) Y_{2}\left(\frac{T}{2}\right)=I_{k}
$$

which is a contradiction.

With (3) we have

$$
Y(T)-I_{2 k}=\left[\begin{array}{cc}
-2 Z_{12} Y_{1}^{\prime}\left(\frac{T}{2}\right) & -2 Z_{12} Y_{2}^{\prime}\left(\frac{T}{2}\right) \\
-2 Z_{21} Y_{1}\left(\frac{T}{2}\right) & -2 Z_{21} Y_{2}\left(\frac{T}{2}\right)
\end{array}\right] .
$$

From this and (6) we get

$$
\operatorname{det}\left(Y_{1}(T)-I_{k}\right)=\operatorname{det}\left(-2 Z_{12} Y_{1}^{\prime}\left(\frac{T}{2}\right)\right)=2^{k} \operatorname{det} Y_{2}\left(\frac{T}{2}\right) \cdot \operatorname{det} Y_{1}^{\prime}\left(\frac{T}{2}\right)
$$

which was the equality in part (i). The rest of the proof can be made in the same way.

Theorem 4 (i) tells us in connection with Theorem 3 that a nontrivial $T$-periodic solution of (1) exists if and only if one of the determinants $\operatorname{det} Y_{1}^{\prime}\left(\frac{T}{2}\right)$ or $\operatorname{det} Y_{2}\left(\frac{T}{2}\right)$ vanishes. Part (ii) can be interpreted analogously. The following lemma characterizes the different cases where periodic/antiperiodic solutions of (1) exist. For $k=1$ this lemma can be found in $[10]$, for instance.

Lemma 2. Equation (1) has an

a) even T-periodic solution if and only if $\operatorname{det} Y_{1}^{\prime}\left(\frac{T}{2}\right)=0$.

b) odd T-periodic solution if and only if $\operatorname{det} Y_{2}\left(\frac{T}{2}\right)=0$.

c) even T-antiperiodic solution if and only if $\operatorname{det} Y_{1}\left(\frac{T}{2}\right)=0$.

d) odd T-antiperiodic solution if and only if $\operatorname{det} Y_{2}^{\prime}\left(\frac{T}{2}\right)=0$.

Proof. a) Each even solution $y(x)$ can be written in the form $Y_{1}(x) c$ with some $c \in$ $\mathbb{C}^{k} \backslash\{0\}$. The periodicity of $y$ implies

$$
Y_{1}(T) c=c \text { and } Y_{1}^{\prime}(T) c=0 .
$$


With equation (2) in Theorem 1 we get

$$
2 Y_{1}^{\prime}\left(\frac{T}{2}\right) c=Y_{1}^{\prime}\left(\frac{T}{2}\right)\left(Y_{1}(T)+I_{k}\right) c=Y_{2}^{\prime}\left(\frac{T}{2}\right) Y_{1}^{\prime}(T) c=0
$$

and therefore $\operatorname{det} Y_{1}^{\prime}\left(\frac{T}{2}\right)=0$. On the other hand, from (8) and

$$
Z_{21} Y_{1}\left(\frac{T}{2}\right)=-Z_{22} Y_{1}^{\prime}\left(\frac{T}{2}\right)
$$

we see that any nontrivial $c$ with $Y_{1}^{\prime}\left(\frac{T}{2}\right) c=0$ defines an even $T$-periodic solution $Y_{1}(x) c$ of $(1)$.

The other statments are proved similarly, for the proof of c) and d) we use the identity

$$
Y(T)+I_{2 k}=\left[\begin{array}{rr}
2 Z_{11} Y_{1}\left(\frac{T}{2}\right) & -2 Z_{12} Y_{2}^{\prime}\left(\frac{T}{2}\right) \\
-2 Z_{21} Y_{1}\left(\frac{T}{2}\right) & 2 Z_{22} Y_{2}^{\prime}\left(\frac{T}{2}\right)
\end{array}\right] .
$$

Final remarks. As we have seen, the Floquet exponents of (1) can be calculated by (numerical) integration either on the interval $\left[0, \frac{T}{2}\right]$ with $2 k$ different initial values or on the interval $[0, T]$ with $k$ different initial values (cf. Remark 1 and Theorem 3 , respectively). A completely different way of computing the Floquet exponents was introduced by HILL himself, using infinite determinants. In [3] we consider infinite determinants corresponding to the matrix form of Hill's equation and prove some fundamental results that enables us to use the determinantal method in the case of arbitrary $k$.

\section{References}

[1] E. Adams, D. Cordes, H. Keppler: Enclosure Methods as Applied to Linear Periodic ODE's and Matrices. Z. angew. Math. Mech. 70 (1990) 12, 565 - 578

[2] L. Cesari: Asymptotic Behavior and Stability Problems in Ordinary Differential Equations. SpringerVerlag, Berlin 1963

[3] R. Denk: Die Determinantenmethode zur Bestimmung der charakteristischen Exponenten von Hillschen Differentialgleichungs-Systemen. Thesis, Universität Regensburg 1993

[4] K. O. Friedrichs: Advanced Ordinary Differential Equations. Nelson, London 1965

[5] G. W. Hill: On the Part of the Motion of the Lunar Perigee which is a Function of the Mean Motions of the Sun and the Moon. Acta Math. 8 (1886), 1 - 36

[6] M. Koecher: Lineare Algebra und analytische Geometrie. Springer-Verlag, Berlin 1983

[7] W. Magnus, S. Winkler: Hill's Equation. Interscience Publishers, New York 1966

[8] J. Meixner, F. W. Schäfke: Mathieusche Funktionen und Sphäroidfunktionen. Springer-Verlag, Berlin 1954

[9] W. T. ReID: Ordinary Differential Equations. John Wiley \& Sons, New York 1971 
[10] F. W. SснӓFKE: Über die Stabilitätskarte der Mathieuschen Differentialgleichungen. Math. Nachr. 4 (1951), $175-183$

[11] B. Weyn: Calculations of Stability Maps of Time-varying Systems. Twelfth Biennial ASME Conference on Mechanical Vibration and Noise (1989) in Montreal, Canada

[12] V. A. Yakubovich, V. M. Starzhinskir: Linear Differential Equations with Periodic Coefficients. J. Wiley \& Sons, New York 1975

\footnotetext{
Universität Regensburg

NWF I - Mathematik

D-93040 Regensburg

Germany
} 